\title{
Portable instrument employing a surface acoustic wave sensor with a regenerable reagent coating for direct measurement of 1,3-butadiene and styrene
}

\author{
Guo-Zheng Zhang and Edward T. Zellers \\ Department of Environmental and Industrial Health, School of Public Health, University of Michigan, 1420 \\ Washington Heights, Ann Arbor, Michigan 48109-2029.
}

(Reccivcd 8 August 1994; accepted for publication 21 September 1994)

\begin{abstract}
The design and preliminary laboratory testing of a prototype portable instrument capable of near-real-time measurement of selected airborne olefins and dienes at low- and sub-ppm concentrations is described. The sensor employed is a $60-\mathrm{MHz}$ surface-acoustic-wave (SAW) oscillator coated with a Pt-olefin coordination compound having the formula trans $-\mathrm{PtCl}_{2}$ (olefin)(pyridine) (olefin $=1$-hexene or ethylene). The continual mass change that occurs upon replacement of the initially coordinated olefin by the olefin or diene analyte leads to a commensurate change in the output frequency of the SAW sensor. Analyte air concentrations can be determined therefore by measuring the rate of frequency change. In tests with 1,3-butadiene and styrene the instrument provided linear response isotherms from 0.050 to 3.0 and 0.60 to $50 \mathrm{ppm}$, respectively, and calculated detection limits of 0.043 and $0.28 \mathrm{ppm}$, respectively, based on a $10 \mathrm{~s}$ measurement interval. Repeated regeneration of the reagent sensor coating is accomplished automatically by brief exposure to a high concentration of the initially coordinated olefin generated from a reservoir that can be housed within the instrument. (C) 1995 American Institute of Physics.
\end{abstract}

\section{INTRODUCTION}

\section{A. Overview}

Coated surface-acoustic-wave (SAW) sensors can provide sensitive and selective measurement of a wide range of gases and vapors. ${ }^{1,2}$ The SAW sensor operates by generating radio-frequency mechanical waves along the surface of a piezoelectric substrate that is coated with a thin layer of a chemically sensitive material. Physical or chemical interactions between the sensor coating and gas-phase analytes lead to changes in the properties of the acoustic waves (e.g., amplitude, phase, or frequency) that can be correlated with the concentration of the analyte in the atmosphere.,

Sensitivity and selectivity are determined largely by the nature of the sensor coating material. Useful SAW sensors have been developed employing either sorptive-polymer coatings or reagent coatings. ${ }^{1}$ In the former case, responses depend on transient changes in the coating mass and elastic stiffness accompanying reversible vapor partitioning. ${ }^{5,6}$ In the latter case, responses depend on the mass change, or the rate of mass change, associated with the chemical reaction between the coating and analyte.

As this sensor technology has matured, reports have begun to appear on field and laboratory testing of prototype portable instruments based on SAW sensors or sensor arrays that use polymeric sensor coatings. ${ }^{8}{ }^{10}$ Portable instrumentation designed for SAW sensors that employ reagent coatings, however, has not been reported.

In a series of recent articles we explored the use of reagents of the general formula trans- $\mathrm{PtCl}_{2}$ (olefin)(amine) as SAW sensor coatings for the measurement of various olefins. ${ }^{7,11-14}$ The olefin ligands in these reagents are bound to the central $\mathrm{Pt}$ atom through $\pi$-orbital coordination and they can be displaced by other olefins under mild conditions according to the following representative equation:

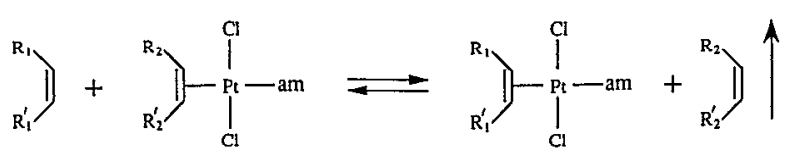

Responses of SAW sensors coated with members of this class of reagents are dependent upon the rate of change of mass accompanying exchange of the free olefin for the initially coordinated olefin in the reagent. The high degree of selectivity observed for certain olefins in the presence of other olefin and nonolefin interferences has been shown to depend on both the steric and electronic properties of the free and bound olefins and the amine ligand coordinated to $\mathrm{Pt}^{13,14}$ We have also shown that butadiene can be measured with this approach, using trans $-\mathrm{PtCl}_{2}\left(1\right.$-hexene)(pyridine). ${ }^{15}$ In this case, however, reaction leads to the substitution of two 1-hexene molecules for each butadiene and formation of the bridged coordination compound $\left[\mathrm{PtCl}_{2} \text { (pyridine) }\right]_{2}$ (butadiene). The continual loss of mass accompanying this reaction is the basis for detection.

In addition to the high sensitivity and selectivity obtained, another useful aspect of this class of SAW sensor coatings is the capability for post-exposure regeneration, in situ: bricf treatment with a high concentration of the initially coordinated olefin drives the reaction shown above in the reverse direction and leads to recovery of the original reagent, thereby extending the service life of the sensor.

In this article we report on the design of a prototype field-monitoring instrument that employs such a coated SAW sensor and on preliminary laboratory results demonstrating direct measurement of low concentrations of butadiene and styrene as well as automatic regeneration of the sensor coatings. 


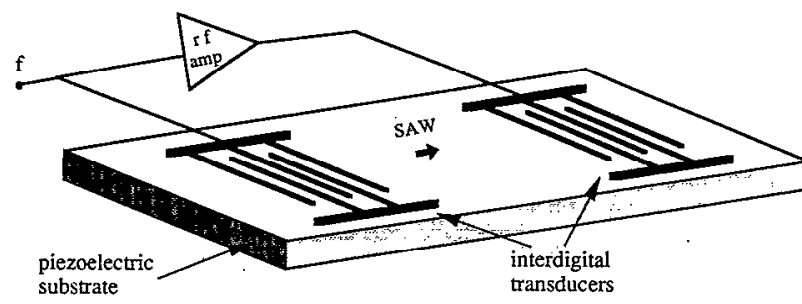

FIG. 1. SAW feedback oscillator configuration used to measure output frequency, $f$.

\section{B. Sensor operating principle}

When a SAW device is used as the frequency controlling element in feedback oscillator circuit (Fig. 1), the oscillation frequency of the device changes in proportion to changes in mass applied to, or removed from, the surface according to the following approximate expression: ${ }^{3}$

$$
\Delta f=(k / a) f_{o}^{2} \Delta m,
$$

where $\Delta f$ is the frequency shift $(\mathrm{Hz}), k$ is a negative substrate constant equal to $-1.26 \mathrm{~cm}^{2} \mathrm{~s} / \mu \mathrm{g}$ for ST-quartz, $a$ is the active area of the SAW device $\left(\mathrm{cm}^{2}\right), f_{o}$ is the sensor operating frequency $(\mathrm{MHz})$, and $\Delta m$ is the mass change $(\mu \mathrm{g})$.

Deposition of a thin reagent coating film onto the SAW device thus lowers the operating frequency. Provided that the gain of the feedback amplifier is high enough, a new stable frequency, $f^{\prime}$, is established. In most cases, a second reference oscillator is used and the difference frequency between coated and reference sensors is measured after mixing signals from each device.

Exposure of the coated sensor to a reactive analyte will generally lead to a change in the mass of the coating which, in turn, will cause a change in $f^{\prime}$. The following equation can then be used to relate the rate of change of frequency to the rate of mass change:

$$
d f^{\prime} / d t=S d m / d t
$$

where the sensitivity factor $S$ increases roughly as the square of the sensor operating frequency. ${ }^{15}$

For the reagents considered in this study, the change of mass associated with the olefin substitution reaction depends on the difference in the molecular weights of the incoming and leaving olefins and on the stoichiometry of the reaction. Since the rate of reaction, and hence the rate of mass change, is a function of the air concentration of the analyte gas or vapor, the rate of frequency change can be related to the instantaneous analyte concentration.

\section{INSTRUMENTATION}

\section{A. General description}

The prototype instrument, constructed in collaboration with Microsensor Systems, Inc. (Bowling Green, KY) according to our design specifications, has overall dimensions of $34 \mathrm{~cm}(d) \times 21 \mathrm{~cm}(w) \times 14 \mathrm{~cm}(h)$ and weighs about $5 \mathrm{~kg}$. Figures 2 and 3 provide block diagrams of the primary pneumatic and electronic system components of the instrument. It

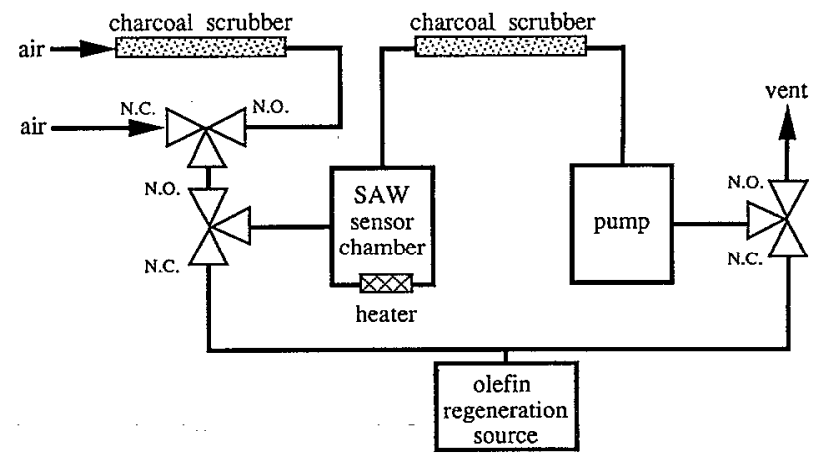

FIG. 2. Block diagram of the pneumatic system components of the prototype instrument $(\mathrm{NO} .=$ normally open; $\mathrm{NC} .=$ normally closed $)$.

contains two matched $60 \mathrm{MHz}$ SAW delay-line oscillators enclosed in a rectangular stainless-steel chamber that is mounted on a printed circuit board together with other signal-conditioning circuitry. A second board supports the frequency counter and pneumatic relays, and a third board contains the microcomputer and an external EPROM chip. A keypad and an LCD readout panel are mounted on the front of the instrument. Three Teflon ${ }^{\circledR}$ solenoid valves (model 225T011, NResearch, Inc., Northboro, MA) are used to direct airflow that is drawn by a small diaphragm pump (model PV-1000, Delta Power Corp., Mashpee, MA) at a rate of 250 $\mathrm{cm}^{3} /$ min through the sensor chamber. Power can be supplied either from an external regulated dc power supply or an onboard $6.3-\mathrm{V} 8 \mathrm{amp} \mathrm{h}$ rechargeable battery. Additional components include a resistive heater, activated-charcoal scrubbers, and a port for introducing the regeneration olefin to the system.

\section{B. Sensor design and mounting configuration}

The SAW devices were fabricated on ST-quartz at the University Michigan Solid-State Electronics Facility. The in-

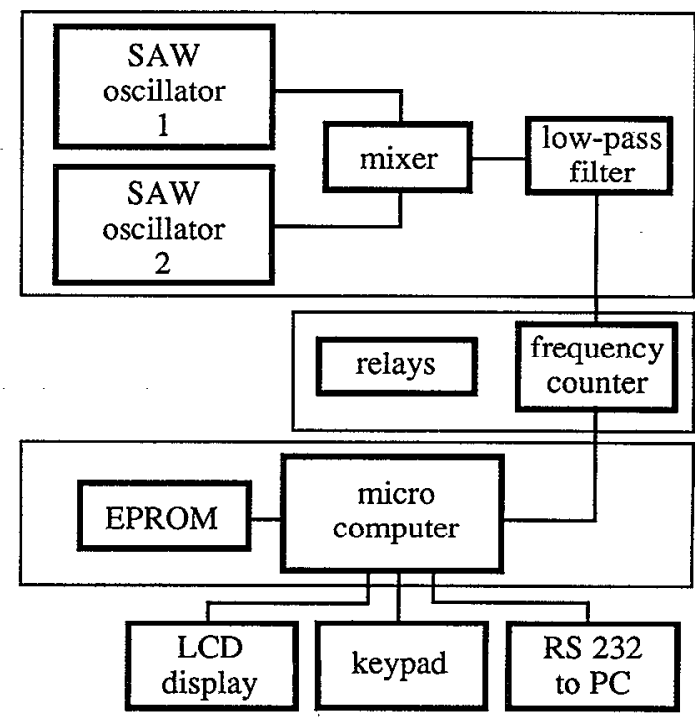

FIG. 3. Block diagram of the primary electronic system components of the prototype instrument. 
terdigital transducers (IDTs) used to launch and receive the acoustic waves consist of 50 pairs of evaporated Au-on-Cr microelectrodes having equal widths and spaces of $13 \mu \mathrm{m}$ and an acoustic aperture of $0.27 \mathrm{~cm}$. The distance between IDT centers is $1.2 \mathrm{~cm}$ yielding an active device area of 0.36 $\mathrm{cm}^{2}$.

The sensors were mounted in parallel inside the stainless-steel chamber $\left(\sim 4 \mathrm{~cm}^{3}\right.$ internal volume). The tophalf of the chamber is equipped with gas inlet and outlet fittings and is sealed to the bottom section with a Teflon(3) gasket and four bolts. The bottom section of the chamber contains a series of holes press fit with Delrin(B) plugs through which integrated-circuit (IC) pins were inserted for making electrical connections between the sensors and the external circuitry. A matching set of IC sockcts soldered onto a printed circuit board beneath the chamber permits the entire chamber to be inserted and removed easily.

A 7 mil layer of Sil-Pad $400^{\circledR}$ (Bergquist, Edina, MN) was placed between the sensors and the floor of the chamber to improve thermal contact, and the sensors were secured in place with the soldered leads connecting the devices to the IC pins around the inner periphery of the chamber. One thermocouple (3 mil diam, chrome-alumel, Omega Engineering, Inc., Stamford, CT) was sandwiched tightly between one of the sensors and the underlying Sil-Pad layer and another was placed in contact with the upper surface of the second sensor. The thermocouple outputs were monitored with separate temperature meters (model HH-71K1, Omega Engineering, Inc.) and were calibrated against an NBS traceable thermometer over the temperature range of interest prior to insertion in the system.

A resistive heater epoxied into a recessed cavity on the outside of the bottom section of the chamber is used for elevated temperature operation. The heater is designed to provide a surface temperature of $65^{\circ} \mathrm{C}$ and draws current to maintain this set point. Accounting for some degree of conductive and convective losses, the heater was expected to provide a temperature of $\sim 40^{\circ} \mathrm{C}$ at the surface of each SAW device.

Temperature monitoring was not incorporated as an intcgral feature of this prototype. In the case of butadiene, our previous studies showed that sensor responses were not affected by temperature in the range of $25-35^{\circ} \mathrm{C} .{ }^{15}$ Thus, precisely maintaining a preset temperature was not necessary within this range and operating without active temperature control was deemed feasible. Still, it is important for the sensor temperature to be relatively constant over the measurement period to minimize thermal baseline drift caused by the inherent temperature sensitivity of the SAW device, but this issue was addressed by using a short measurement cycle and subtracting any pre-exposure baseline drift from subsequent readings. The large thermal mass of the exposurc chamber also helps to buffer any abrupt changes in the ambient temperature. For styrene, the sensor response has been shown to exhibit a positive Arrhenius temperature dependence, and maintaining a constant sensor temperature is more important. At $40^{\circ} \mathrm{C}$, a limit of detection (LOD) of $\sim 1$ $\mathrm{ppm}, \mathrm{v} / \mathrm{v}$ was expected according to previous studies. ${ }^{11} \mathrm{~A}$ slightly higher or lower LOD was acceptable, however, so once again an exact set point for the temperature was not critical.

\section{Pneumatics}

Three modes of operation are used corresponding to three different valve settings and flow paths (Fig. 2). In base line mode, incoming air is passed through the activatedcharcoal inlet scrubber, then sequentially through the chamber, the in-line scrubber, the pump, and then to exhaust. In sample mode, incoming air bypasses the inlet scrubber and then follows the same path as that traced in base line mode. In regeneration mode, a closed loop is created by switching the inlet valve and the valve connecting the regeneration reservoir to the sensor chamber. Air is then circulated past the reservoir, through the sensor chamber, and then through the in-line scrubber and pump.

Tests with butadiene were performed using coatings of trans- $\mathrm{PtCl}_{2}$ (1-hexene)(pyridine) and tests with styrene were performed using coatings of trans- $\mathrm{PtCl}_{2}$ (ethylene)(pyridine). For the former, a $4 \mathrm{~mL}$ septum-topped vial containing a small section of polyurethane foam soakcd in 1-hexene was used as the regeneration source. The septum was pierced with a $1 / 8$-in. diam stainless steel tube connected to the system with a tee fitting. For the latter tests, a separate lecture bottle of ethylene equipped with a regulator and flow restrictor was used for regeneration via the same fitting.

\section{Signal processing, data acquisition, and data management}

The electronic components of the instrument are distributed between three printed circuit boards. The amplifiers and inductive tuning elements used to condition the individual sensor signals, as well as the double-balanced mixer and low-pass filter used to obtain the difference frequency between the coated and reference sensors, are all located on the board upon which the sensor chamber is mounted. Signals are tapped from this circuit and passed to the frequency counter located on a separate board and subsequently to the microcomputer/EPROM board. Since the difference frequencies are typically in the range of $100-400 \mathrm{kHz}$, radiofrequency interference is not a problem and shielding of the signal transfer circuit is not necessary.

An Intel $8052 \mathrm{AH}$ microcomputer having $8 \mathrm{~K}$ of program memory, $8 \mathrm{~K}$ of data memory, and an external $64 \mathrm{~K}$ EPROM is used for instrument control. The difference frequency signals are converted to air concentration values and displayed on the $2 \times 12$-digit LCD on the front panel. Concentration values are periodically stored in battery-backed, nonvolatile RAM and subsequently transferred to a personal computer through an RS-232 communication port on the backpanel of instrument. The program used for instrument control and data acquisition/management was written in MCS-52 BASIC. It occupies approximately $6 \mathrm{~K}$ of EPROM memory and is automatically executed upon powering the instrument.

The LCD is used to relay instructions to the operator. From the main menu, the operator can select one of three functions (MODIFY, DATA DUMP, and RUN) by simple keypad entry. The MODIFY option provides a display of the 
current settings for the duration of each operational mode (i.e., baseline, sample, or regeneration) and allows these to be modified as needed. The DATA DUMP option initiates the transfer of data as an ASCII file from the instrument RAM to the personal computer. The RAM can also be cleared of the current data log for subsequent data storage. The RUN option initiates data collection and solenoid-valve actuation, and allows the operator to select an individual operating mode or to initiate a cycle whereby the modes are executed in automatic sequence.

In baseline mode, the difference frequency from the sensors is displayed in $\mathrm{Hz}$ and is updated every $10 \mathrm{~s}$. The average baseline drift, if any, is calculated and stored. In sample mode, the air concentration of the analyte is displayed in $\mathrm{ppm}$. Concentration values are determined from the net frequency response over the sampling interval by first subtracting any baseline drift and then multiplying by a conversion factor obtained from calibration. The upper line of the LCD updates the analyte concentration every few seconds and the lower line of the display shows the 1 min average concentration. The latter values are stored in RAM along with a time-indexed sample number so that a record of concentration versus time can be retrieved at the conclusion of sampling. The RAM is capable of storing up to 1120 records of 5 bytes each, corresponding to approximately $18 \mathrm{~h}$ of data collection. During regeneration, difference frequency readings in $\mathrm{Hz}$ are displayed and updated every $10 \mathrm{~s}$ but are not stored.

\section{EXPERIMENTAL METHODS}

All solvents and reagents were obtained from the Aldrich Chemical Co. (Milwaukee, WI) and were used as received. The trans $-\mathrm{PtCl}_{2}$ (1-hexene)(pyridine) and trans $-\mathrm{PtCl}_{2}$ (ethylene)(pyridine) reagents were synthesized from Ziese's salt by known methods. ${ }^{16}$ The former is highly sensitive to butadiene gas and insensitive to styrene, and the latter is sensitive to styrene but not to butadiene. 15 trans $-\mathrm{PtCl}_{2}$ (ethylene)(pyridine) forms yellow needlelike crystals and can be stored for more than a year without decomposition. The trans- $\mathrm{PtCl}_{2}$ (1-hexane)(pyridine) is generally recovered as a viscous oil, although it can be isolated as a low-melting solid $\left(\mathrm{mp}=40^{\circ} \mathrm{C}\right)$ by repeated recrystallization from hexane/methylene chloride. This reagent has shown evidence of a loss of activity after about 2-3 months of storage at room temperature, so only freshly synthesized material was used for testing. The purities of the reagents and the corresponding products of reaction were confirmed by elemental analysis (Galbraith Laboratories, Knoxville, TN).

Combining the reagents with the rubbery amorphous polymer, polyisobutylene (PIB), has been shown to improve coating uniformity and adhesion. ${ }^{7}$ Solutions of approximately $6 \mathrm{mg} / \mathrm{mL}$ of each component in 2:1 toluene:hexane are applied to one of the SAW devices using an air brush until a frequency shift in the range of $150-400 \mathrm{kHz}$ is observed. Since the mass sensitivity of the $60-\mathrm{MHz}$ SAW device is approximately $12.6 \mathrm{kHz} / \mu \mathrm{g}$ [from Eq. (1)], this range of coating frequency shift values corresponds to deposited masses of approximately 16-32 $\mu \mathrm{g}$. Further addition of coat-

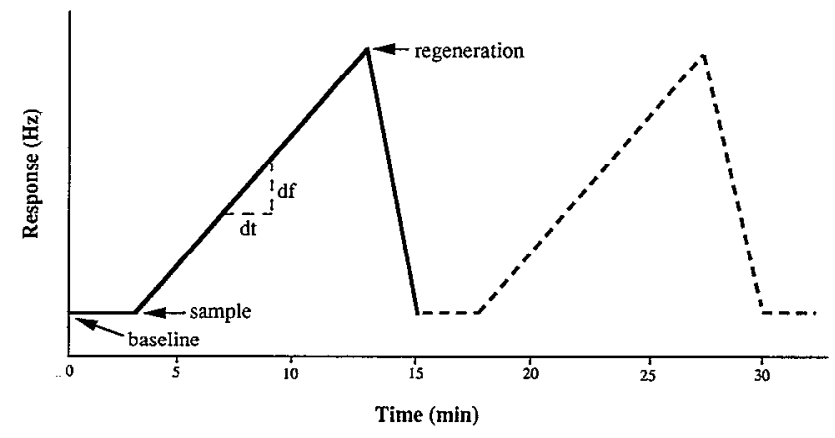

FIG. 4. Typical base line-sample-regeneration test cycle.

ing generally results in a loss of oscillation due to insufficient amplifier gain. After coating, the sensor is allowed to stand for at least $2 \mathrm{~h}$ to allow the solvent to evaporate and the baseline to stabilize.

For most of the tests performed, the reference oscillator was coated with PIB in order to cancel any mass changes arising from sorption of vapors into the PIB component of the working sensor coating. For butadiene, this is not necessary because butadiene does not partition to any measurable extent into the PIB at the concentrations of interest. ${ }^{15}$ However, for styrene and several potentially interfering solvent vapors, the partitioning would be greater. In situations where there are fluctuating concentrations of these vapors, the signal due to sorption into the PIB would be superimposed on the signal due to reaction of the target analyte with the reagent. Such signals were effectively masked by applying equivalent amounts of PIB to the working and reference devices.

Test atmospheres were prepared by injection of styrene liquid or butadiene gas into a $10 \mathrm{~L}$ Tedlar@ bag followed by dilution with clean air that was metered into the bag with a precision dry-gas meter. Aliquots were withdrawn with a gas-tight syringe, injected into each of several additional bags, and diluted as necessary to obtain atmospheres spanning a range of lower concentrations. The concentrations in the bags were confirmed with a calibrated photoionization detector (model PI-101, HNu Systems, Newton, PA).

The instrument was placed in a laboratory exhaust hood for all exposure tests. At least 30-60 min was required after powering up for temperature stabilization in the sensor chamber. Experiments were performed initially with the program running from a personal computer. The program was modified to allow monitoring of both the frequency and the calculated air concentrations during sample mode. Subsequent tests were performed with the program downloaded to the instrument microcomputer. Figure 4 shows a typical test cycle profile using durations of $3 \mathrm{~min}$ for baseline, $10 \mathrm{~min}$ for sampling and 2 min for regeneration. At the highest concentrations tested for both styrene and butadiene, the sample duration was reduced since responses were high enough that significant depletion of the reagent, and consequent reduction in responses, occurred within a few minutes of exposure. 


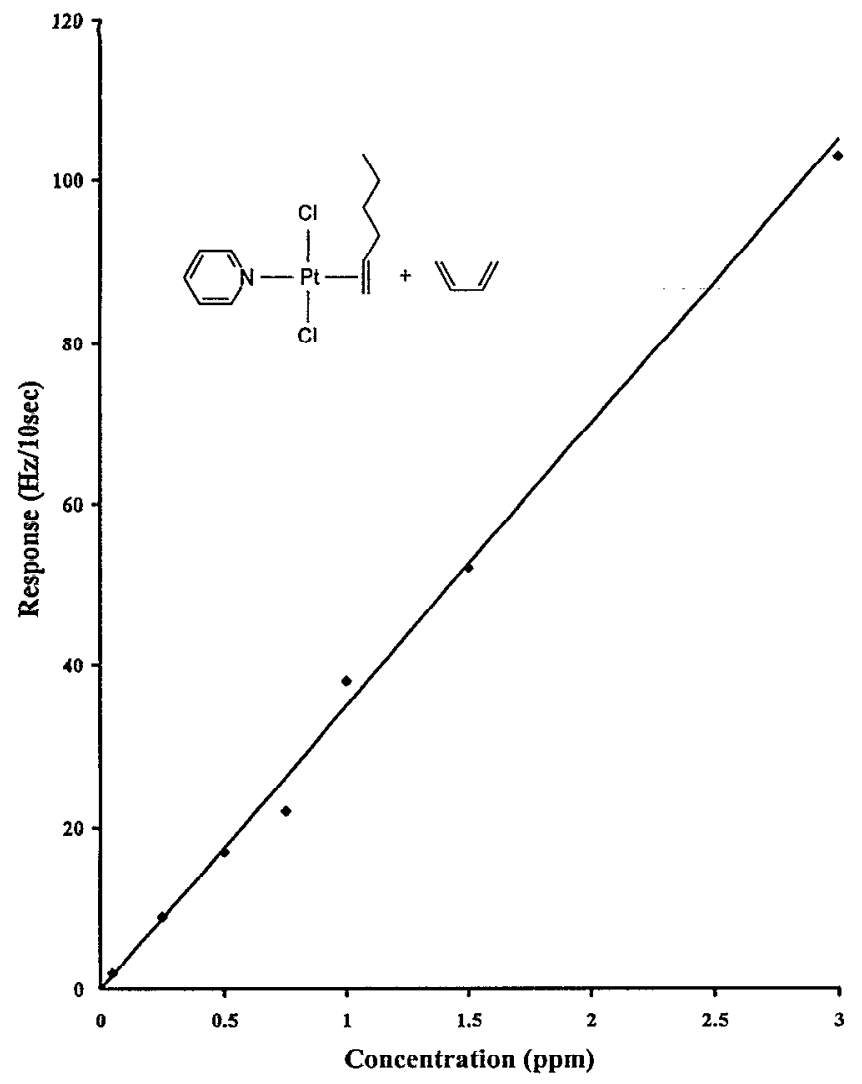

FIG. 5. Calibration curve for butadiene using a sensor coating of trans- $\mathrm{PtCl}_{2}$ (1-hexene)(pyridine) + PIB.

\section{RESULTS AND DISCUSSION}

\section{A. Mode cycling and temperature stability}

The instrument was designed to read the difference frequency from the SAW devices at $2 \mathrm{~s}$ intervals. However, somewhat more time was required for conversion of the frequency readings to air concentration values and subsequent display. As a result, the minimum time for updating the display was $4 \mathrm{~s}$. This does not represent an important limitation, however, since such rapid measurements were not necessary. For convenience, an interval of $10 \mathrm{~s}$ was used for most of the tests performed.

The instrument was cycled through baseline, sample, and regeneration modes continuously with and without the heater activated with clean air passing over the sensors. Without the heater, the temperature within the chamber ranged from 26 to $28^{\circ} \mathrm{C}$ over several hours and was consistently about $2-3^{\circ} \mathrm{C}$ above room temperature. With the heater activated the sensor temperature ranged from 41 to $43^{\circ} \mathrm{C}$ over several hours, and also showed a slight dependence on the ambient temperature. The thermocouples located on the upper and lower device surfaces gave the same readings at all times. The temperature stability was reflected in the stability of the output frequency which typically changed by less than $1-2 \mathrm{~Hz}$ between successive $10 \mathrm{~s}$ measurements over the $10 \mathrm{~min}$ sampling period at either operating temperature.

To probe the temperature sensitivity further, the temperature was ramped from 26 to $38^{\circ} \mathrm{C}$ over the course of $20 \mathrm{~min}$

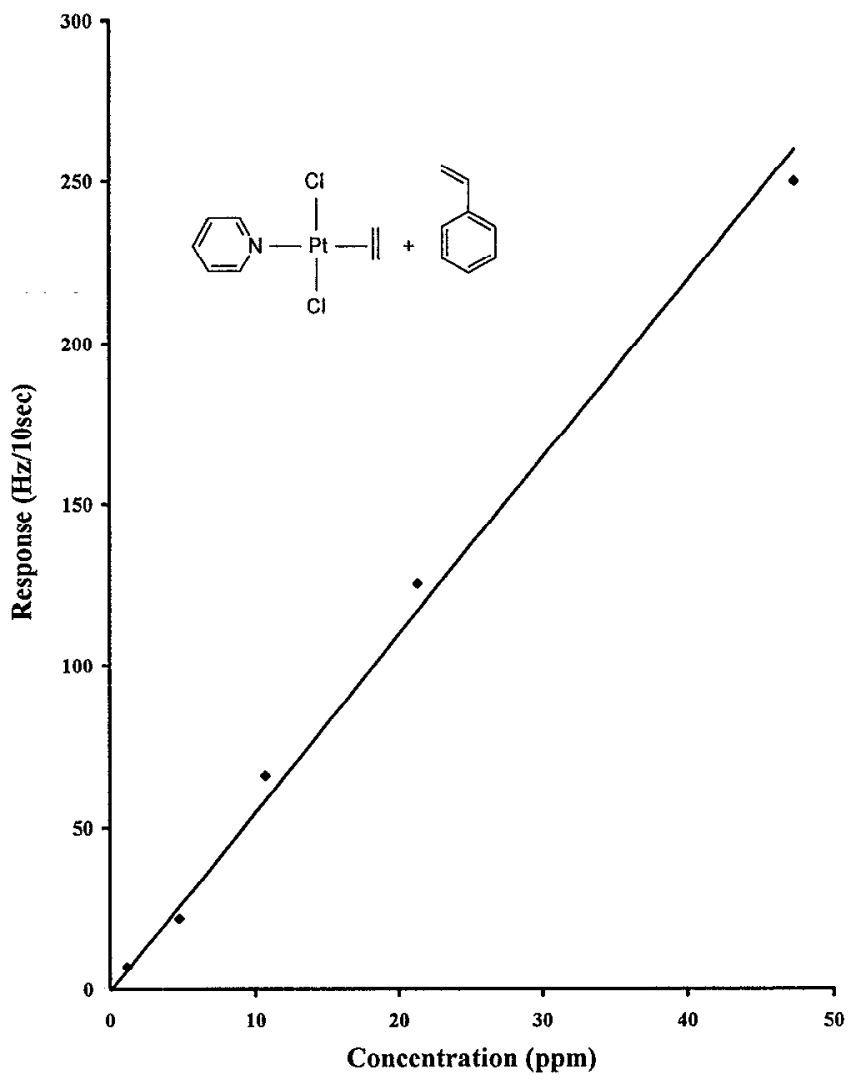

FIG. 6. Calibration curve for styrene using a sensor coating of trans- $\mathrm{PtCl}_{2}$ (ethylene)(pyridine) $+\mathrm{PIB}$.

with clean air passing through the system. The plot of temperature versus the difference frequency was linear $\left(r^{2}=0.979\right)$ and gave a slope of $163 \mathrm{~Hz} /{ }^{\circ} \mathrm{C}$. This temperature coefficient is significantly lower than those reported by other researchers for individual polymer-coated ST-quartz SAW sensors operating at higher frequencies. ${ }^{17}$ As stated above, during operation any temperature-induced drift is accounted for by storing the pre-exposure baseline frequencies and subtracting them from the sample-mode readings.

Switching from regeneration mode to baseline mode consistently gave a large spikc in the output signal owing apparently to a power surge associated with actuating the solenoid valves. A similar, though smaller spike was observed on switching from baseline to sample mode. To remove the effect of this artifact on concentration determinations, the first 10-s reading following switching modes had to be disregarded and was automatically omitted from the calculation of $1 \mathrm{~min}$ average readings.

\section{B. Calibration curves for butadiene and styrene}

Butadiene is used primarily as a feedstock chemical for the production of polymer resins and elastomers. It is classified as a suspected carcinogen, as well as a possible teratogen and reproductive hazard. ${ }^{18,19}$ The U. S. Occupational Safety and Health Administration (OSHA) has recently proposed a reduction in the $8 \mathrm{~h}$ average permissible exposure limit (PEL) for butadiene from the current value of 1000 to 2 
ppm, with a proposed action level of $1 \mathrm{ppm}$. Thus, our goal was to measure butadiene in the vicinity of the proposed OSHA standards.

Figure 5 shows a calibration curve for butadiene obtained at $26^{\circ} \mathrm{C}$ using a $230 \mathrm{kHz}$ coating of trans- $\mathrm{PtCl}_{2}$ (1-hexene)(pyridine). Each point on the graph represents the average $10 \mathrm{~s}$ response at the indicated concentration. The rate of frequency change is linearly related to the butadiene concentration from 0.050 to $3.0 \mathrm{ppm}$ and the sensitivity is $34.4 \mathrm{~Hz} / \mathrm{ppm}$ as determined from the slope of the curve $\left(r^{2}=0.997\right)$. Standard deviations $(s)$ around the $1 \mathrm{~min}$ mean steady-state rate of frequency change at a given exposure concentration ranged from $0.5 \mathrm{~Hz}$ at low concentrations (i.e., $<0.50 \mathrm{ppm}$ ) to only $2 \mathrm{~Hz}$ at high concentrations (i.e., $>2$ $\mathrm{ppm})$. This corresponds to uncertainties in concentration determinations of $<10 \%$ over most of the calibrated range. From these data a calculated detection limit of $0.043 \mathrm{ppm}$ in $10 \mathrm{~s}$ is obtained using a criterion of $3 s$ /sensitivity as the minimum detectable signal. The response time was $<10 \mathrm{~s}$, which is comparable to the theoretical mixing time for the chamber and indicates rapid attainment of steady-state conditions within the coating film.

Styrene is also used primarily for the production of polymers. The current OSHA PEL for styrene is $50 \mathrm{ppm},{ }^{20}$ however, evidence of carcinogenicity ${ }^{21}$ suggests that exposures be maintained well below the PEL value. Figure 6 shows a typical calibration curve for styrene at $42{ }^{\circ} \mathrm{C}$ using a $255 \mathrm{kHz}$ coating of $\mathrm{PtCl}_{2}$ (ethylene)(pyridine). Once again, each point on the graph represents the average $10 \mathrm{~s}$ response at the indicated concentration. The sensor response is linear over the measured range of $0.60-50 \mathrm{ppm}$ and the sensitivity is $5.28 \mathrm{~Hz} / \mathrm{ppm}$ (linear regression $r^{2}=0.995$ ). Above $50 \mathrm{ppm}$ the response is so large that a steady value could not be maintained for more than about $2 \mathrm{~min}$. Lower temperature operation could be used to obtain measurements at higher concentrations. Standard deviations around the 1 min mean steady-state rate of frequency change at a given exposure concentration were similar to those for butadiene, and the calculated LOD is $0.28 \mathrm{ppm}$. The time required to reach a constant rate of frequency change (20-30 s) was longer than that for butadiene due to the slower diffusion of styrene into the coating film.

Although this study did not focus on selectivity, our previous work has shown that butadiene can be measured successfully in the presence of high concentrations of butane, ethanol, benzene, hexane, acetone, dichloromethane, or vinylidene chloride. ${ }^{15}$ Similarly, the response to styrene is not affected by exposure to acetone, dichloromethane, toluene, ethylbenzene, methylmethacrylate, vinyl chloride, or acrylonitrile. ${ }^{11}$

\section{Service life and reagent regeneration}

As exposure to either buladiene or styrene proceeds, the reagent in the coating eventually becomes depleted and the sensor response declines. A plot of frequency shift versus time for prolonged exposure to $0.50 \mathrm{ppm}$ of butadiene is shown in Fig. 7. The exposure was continued until no further change in frequency was observed. The ordinate in Fig. 7 has been normalized by dividing the frequency readings by the cumulative frequency shift. Up to approximately $25 \%$ of the total frequency shift (i.e., $3.1 \mathrm{kHz}$ for this coating) the response is linear meaning that the rate of frequency change is constant. Beyond this point the rate declines continuously. Complete conversion to the bridged butadiene-Pt reaction product results in a calculated net loss of $13.3 \%$ of the original mass of the coating. Since the reagent comprises $50 \%$ of the original mass, the total expected frequency shift is, from

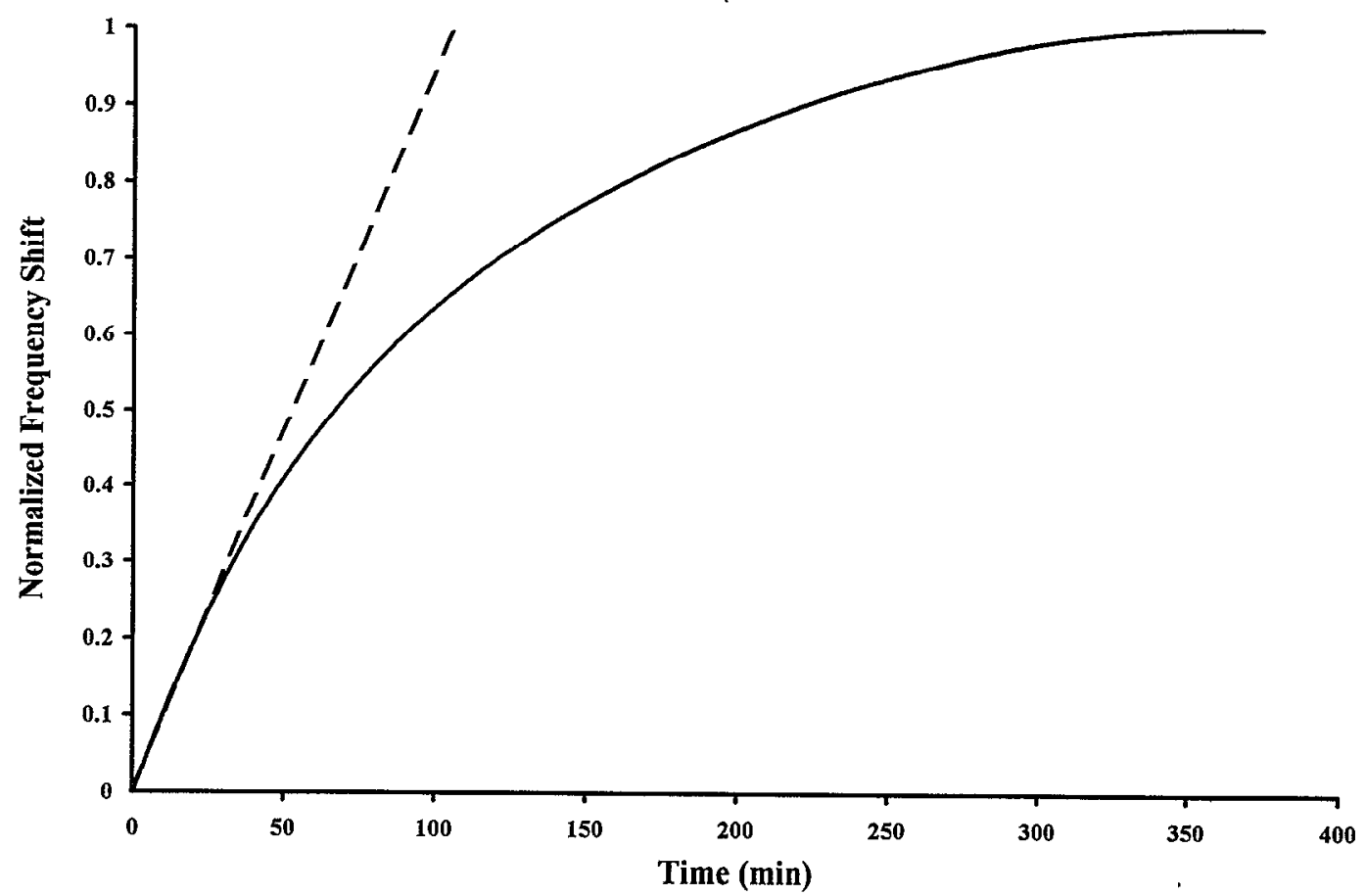

FIG. 7. Plot of fractional frequency shift vs time for exhaustive exposure to butadiene using a $190 \mathrm{kHz}$ coating of trans- $\mathrm{PtCl}_{2}$ (1-hexene)(pyridine) $+\mathrm{PIB}$. 
TABLE I. Regeneration following exhaustive exposure to butadiene and styrene.

\begin{tabular}{|c|c|c|}
\hline & $\begin{array}{c}\text { Butadiene }+ \\
\mathrm{PtCl}_{2}(1 \text {-hexene })(\mathrm{pyr})\end{array}$ & $\begin{array}{c}\text { Styrene }+ \\
\mathrm{PtCl}_{2}(\text { ethylene })(\mathrm{pyr})\end{array}$ \\
\hline Coating freq shift $(\mathrm{kHz})$ & 330 & 294 \\
\hline Mass of reagent $(\mu \mathrm{g})$ & 13.1 & 11.7 \\
\hline Calculated total $\Delta f(\mathrm{~Hz})$ & 21950 & 29700 \\
\hline Experimental total $\Delta f(\mathrm{~Hz})^{\mathrm{a}}$ & $-21197(1420)^{c}$ & $28560(396)^{c}$ \\
\hline Experimental total $\Delta f(\mathrm{~Hz})^{\mathbf{b}}$ & $21318(1132)$ & $-28754(482)$ \\
\hline$\%$ difference ${ }^{d}$ & -3.1 & -3.5 \\
\hline
\end{tabular}

"Total frequency shift for exhaustive exposure.

bTotal frequency shift for regeneration.

Values in parentheses are standard deviations ( $n=5$ for butadiene, $n=3$ for styrene).

${ }^{\mathrm{d} C a l c u l a t e d}$ as $[(\operatorname{expt} \Delta f$-calc $\Delta f) /$ calc $\Delta f] \times 100$, where expt $\Delta f$ is the average absolute $\Delta f$ of the exposure and regeneration cycles.

Eq. (1), $12.64 \mathrm{kHz}$ which matches closely the total shift of $12.40 \mathrm{kHz}$ observed experimentally.

The point at which the response becomes nonlinear defines the effective service life for continuous exposure. For the example shown in Fig. 7, the service life was about 26 min. The lower the butadiene concentration, the longer the service life and vice versa. In addition, the greater the mass of coating deposited, the longer the service life. It should be emphasized, however, that within the predepletion response range the short-term sensor response is independent of the amount of coating deposited. ${ }^{15}$

To examine the regenerability of the trans- $\mathrm{PtCl}_{2}$ (1-hexene)(pyridine) compound, an initial series of exhaustive exposures was performed with a $330 \mathrm{kHz}$ coating using relatively high concentrations of butadiene. At $\sim 100 \mathrm{ppm}$, complete reaction was achieved within a few minutes of exposure as evidenced by no further frequency change. Introduction of 1-hexene vapor caused a rapid recovery of the frequency. The sensor was then exposed and regenerated four more times under similar conditions. Net frequency shifts of -21.95 and $+21.95 \mathrm{kHz}$ were expected following exhaustive exposure and regeneration, respectively. The average experimental values shown in Table I are in remarkably close agreement with these expected values.

Table II presents results of repeated exposure/ regeneration tests using a $201 \mathrm{kHz}$ coating, an exposure concentration of $0.50 \mathrm{ppm}$ of butadiene and the standard baseline, sample, and regeneration mode durations of 3,10 , and 2 min, respectively. This cycle was repeated 11 times, which corresponds to a total of about $3 \mathrm{~h}$ of operation and just under $2 \mathrm{~h}$ of actual exposure to butadiene. If the regeneration were not successful, a progressive decline in response would have been expected after the second cycle and the reagent would have been completely consumed by the 11 th cycle. The results in Table II demonstrate that the reagent can be repeatedly regenerated. The slight decrease in sensitivity observed is suspected of being due to incomplete scrubbing of the butadiene during regeneration, or possibly to residual 1-hexene being present. It is known that activated charcoal has a rather low capacity for butadiene, ${ }^{22}$ although the amount released from the sensor coating upon regeneration is very small. Displacement of trapped butadiene from the
TABLE II. Sensitivity after multiple exposure/regeneration cycles.

\begin{tabular}{ccc}
\hline & \multicolumn{2}{c}{$\begin{array}{c}\text { Mean response (s.d.) } \\
\mathrm{Hz} / 10 \mathrm{~s}\end{array}$} \\
& \multicolumn{3}{c}{ Butadiene } & $\begin{array}{c}\text { Styrene } \\
+\end{array}$ \\
& + & $\mathrm{PtCl}_{2}$ (ethylene)(pyr) \\
\cline { 2 - 3 } Cycle & $\mathrm{PtCl}_{2}(1$-hexene)(pyr) & $69(3)$ \\
\hline 1 & $-20(1.2)$ & $70(7)$ \\
2 & $-19(0.4)$ & $72(6)$ \\
3 & $-19(0.4)$ & $76(3)$ \\
4 & $-20(0.4)$ & $73(6)$ \\
5 & $-19(0.4)$ & \\
6 & $-18(0.3)$ & \\
7 & $-17(0.5)$ & \\
8 & $-17(0.5)$ & \\
9 & $-18(0.5)$ & \\
10 & $-17(0.5)$ & \\
11 & $-17(0.6)$ & \\
\hline \hline
\end{tabular}

axposure concentrations: butadiene $=0.50 \mathrm{ppm}\left(26^{\circ} \mathrm{C}\right)$; styrene $=10 \mathrm{ppm}$ $\left(41^{\circ} \mathrm{C}\right)$. The values presented were determined from 1 min readings averaged over the $10 \mathrm{~min}$ duration of each cycle.

charcoal surface by the more strongly adsorbed 1-hexene might also be occurring. In any case, the sensitivity is held within $15 \%$ of the initial value for several hours.

Table I also presents the results of exhaustive exposure/ regeneration tests with styrene and trans $-\mathrm{PtCl}_{2}$ (ethylene)(pyridine). Exposure to $1000 \mathrm{ppm}$ of styrene for several minutes was followed by regeneration via brief exposure to a high concentration of ethylene gas. The initial frequency shift due to coating deposition was 294 $\mathrm{kHz}$. From the mass difference between styrene and ethylene and the 1:1 stoichiometry of the substitution reaction, complete conversion would correspond to a net frequency shift of $29.7 \mathrm{kHz}$ or $20.2 \%$. The average experimental values of 28.6 and $-28.8 \mathrm{kHz}$ for triplicate exposure and regeneration tests, respectively, are in close agreement with this value.

Results of similar exposure/regeneration cycling with 10 ppm of styrene are shown in Table II. Without regeneration a decline in response would have been expected during the second exposure cycle and complete consumption would have been expected by the fifth cycle. As shown, the regenerated reagent retains the original sensitivity.

Additional exposure/regeneration tests performed periodically over several days gave mixed results. Reductions in sensitivity were observed in some cases as early as the second day of exposure for both styrene and butadiene. In the case of the trans- $\mathrm{PtCl}_{2}$ (1-hexene)(pyridine) complex we believe the decline may be due, in part, to morphological changes associated with partial crystallization of the reagent over time. This would reduce diffusion of the butadiene into the reagent and would also place steric constraints on the formation of the bridged reaction product. The loss in sensitivity to styrene observed with trans $-\mathrm{PtCl}_{2}$ (ethylene)(pyridine) is less easily explained. This reagent forms crystals directly upon deposition, although the possibility that changes in crystal structure contribute to the loss in sensitivity cannot be ruled out. Neither of the reagents or the products show evidence of chemical decomposition over several weeks of storage. As suggested above, incomplete 
scrubbing of the analytes or regeneration olefins may be contributory.

\section{DISCUSSION}

The results presented here demonstrate that direct measurement of styrene and butadiene at low- and sub-ppm concentrations is possible using the prototype instrument described, and that the service life of the sensor can be extended by automated regeneration of the reagent coatings. The high sensitivity achieved with the relatively lowfrequency $60 \mathrm{MHz}$ SAW oscillators employed here is noteworthy and is attributable to the high rate of the olefinsubstitution reaction between the target analytes and the $\mathrm{PtCl}_{2}$ (olefin)(pyridine) reagents.

Due to the consumption of the reagent over time, there is a need for frequent regeneration. However, only a small amount of the initially coordinated olefin is needed for each regeneration cycle. For regenerating the reagent used to measure butadiene, a small vial housed within the instrument was used which contained enough 1-hexene for several days or even weeks of operation. For regenerating the reagent used to measure styrene, a source of ethylene gas is required. For this study, ethylene was supplied from a separate lecture bottle. In practice a small low-pressure gas bottle containing ethylene in $\mathrm{N}_{2}$ could be used as a safe source of this regenerating agent and could be easily housed within the instrument.

Although this work focused on measuring styrene and butadiene, certain other olefins can also be measured with the coated SAW sensors used here. Thus, extension of this approach to other analytes should be possible.

A number of instrument design modifications are suggested by the results presented above. For example, higher amplifier gain would permit the deposition of more reagent and a corresponding increase in the time interval required between regeneration cycles. Alternatives to the activated charcoal scrubbers need to be considered in order to increase the adsorption capacity for the analytes and the displaced olefins. Granular adsorbents composed of graphitized carbon or carbon molecular sieves, for example, can provide greater capacities for the lower molecular weight species like butadiene and ethylene. ${ }^{23}$ Although temperature control was adequate, a more efficient heating mechanism would reduce power demands which, in turn, would reduce the overall size and weight of the instrument. Finally, further study of medium-term aging effects is needed to determine the factors affecting the loss in sensitivity over time.

\section{ACKNOWLEDGMENTS}

The authors would like to express their appreciation to Terry Hull for assistance in the fabrication of the SAW devices, and to Mingwei Han and several staff members at Microsensor Systems, Inc. for assistance in software development. This work was funded by Grant No. R01-OH02663 from the National Institute for Occupational Safety and Health of the Centers for Disease Control.

${ }^{1}$ M. S. Nieuwenhuisen and A. Venema, Sensors Mater. 5, 261 (1989).

${ }^{2}$ A. D'Amico and E. Verona, Sensors Actuators 17, 55 (1989).

${ }^{3} \mathrm{H}$. Wohltjen, Sensors Actuators 5, 307 (1984).

${ }^{4}$ S. J. Martin, G. C. Frye, and S. D. Senturia, Anal. Chem. 66, 2201 (1994).

${ }^{5}$ J. W. Grate, M. Klusty, R. A. McGill, M. H. Abraham, G. Whiting, and J. Andonian-Haftvan, Anal. Chem. 64, 610 (1992).

${ }^{6}$ S. J. Patrash and E. T. Zellers, Anal. Chem. 65, 2055 (1993).

${ }^{7}$ E. T. Zellers, R. M. White, and S. M. Rappaport, Anal. Chem. 62, 1223 (1990).

${ }^{8}$ G. C. Frye, S. J. Martin, R. W. Cernosek, K. B. Pfeifer, and J. S. Anderson, Proc. IEEE Ultrasonics Symposium, Orlando, FT s, 1991, p. 311.

${ }^{9}$ G. C. Frye, S. J. Martin, R. W. Cernosek, and K. B. Pfeifer, Internat. J. Environ. Conscious Manuf. 1, 37 (1992)

${ }^{10}$ J. W. Grate, S. L. Rose-Pehrsson, D. Venezky, M. Klusty, and H. Wohltjen, Anal. Chem. 65, 1868 (1993).

${ }^{11}$ E. T. Zellers, N. C. Hassold, R. M. White, and S. M. Rappaport, Anal. Chem. 62, 1227 (1990).

${ }^{12}$ E. T. Zellers, Chemical Sensors and Microinstrumentation, ACS Symposium Series 403 , edited by R. W. Murray, R. Seitz, W. Heineman, R. Dessy, and J. Janata (American Chemical Society, Washington, DC, 1989), pp. 176-190.

${ }^{13}$ E. T. Zellers and G. Z. Zhang, Anal. Chem. 64, 1277 (1992):

${ }^{14}$ E. T. Zellers and G. Z. Zhang, Analy. Chim. Acta 280, 1 (1993).

${ }^{15}$ G. Z. Zhang and E. T. Zellers, Anal. Chem. 65, 1340 (1993).

${ }^{16}$ M. Orchin and P. J. Schmidt, Inorg. Chem. Acta Rev. 123 (1968).

${ }^{17}$ J. W. Grate, S. W. Wenzel and R. M. White, Anal. Chem. 64, 413 (1992).

${ }^{18}$ Occupational Safety and Health Administration, Occupational Exposure to 1,3-Butadiene; Proposed Rule and Notice of Hearing, Fedcral Registcr, 29 CFR Part 1910, Vol. 55, No. 155, pp. 32736-32826 (August 10, 1990).

${ }^{19}$ National Institute for Occupational Safety and Health, Current Intelligence Bulletin 41: 1,3 Butadiene, Publ. 84-105, (USDHHS-NIOSH, Cincinnati, OH, 1984), pp. 183-201.

${ }^{20}$ U. S. Department of Labor, Code of Federal Regulations: 29CFR1910.1000 (Department of Labor, Occupational Safety and Health Administration, Occupational Safety and Health Standards, January 19, 1989).

${ }^{21}$ American Conference of Governmental Industrial Hygienists, 1993-1994 $T L V$-Threshold Limit Values and Biological Exposure Indices (ACGIH, Cincinnati, $\mathrm{OH}, 1993)$, p. 32 :

${ }^{22}$ R. A. Lunsford, Y. T. Gagnon, J. Palassis, J. M. Fajen, D. R. Roberts, and P. M. Eller, Appl. Occup. Environ. Hyg. 5, 310 (1990).

${ }^{23}$ Supelco, Inc., GC Bulletin 849C-Efficiently Monitor Toxic Airborne Compounds by Thermal Desorption (Supelco, Inc., Bellefonte, PA, 1988). 